5th ICTA Beam Dynamics Workshop, Corpus Christi, TX, 10/3-8/91.

BNL--46591

BNL

DE92 002088

\title{
ERROR ANALYSIS OF ACCELERATION CONTROL LOOPS OF A SYNCHROTRON *
}

\author{
S.Y. Zhang and W.T. Weng \\ AGS Department, Brookhaven National Laboratory, \\ Upton, New York 11973
}

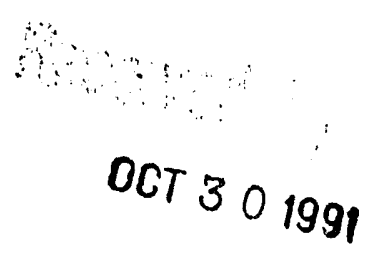

\begin{abstract}
For beam control during acceleration, it is conventional to derive the frequency from an external reference, be it a field marker or an external oscillator, to provide phase and radius feectoack loops to ensure the phase stability, radial position and emittance integrity of the beam. The open and closed loop behaviors of both feedback control and their response under the possible frequency, phase and radius errors are derived from fundamental principles and equations. The stability of the loops is investigated under a wide range of variations of the gain and time delays. Actual system performance of the AGS Booster is analyzed and compared to commissioning experiences. Such analysis is useful for setting design criteria and tolerances for new proton synchrotrons.
\end{abstract}

\subsection{Introduction}

An RF beam control system for a circular accelerator is designed to serve basically two purposes. First, to damp the coherent longitudinal oscillations to avoid emittance blow-up; and secondly, to reduce the particle phase and radial deviations to the minimum.

Acceleration in a synchrotron can be readily achieved if all parameters involved have infinite accuracy and can be realized instantaneously without delay. In reality, many deviations can occur, such as the inaccuracy of the accelerating frequency, the phase and energy errors of the bunched beams at injection, the sensitivity of the RF system components, and finally, the synchrotron oscillation itself. To ensure that the whole process is stable under small deviation of design value, phase and radial feedback loops are introduced.

In this article, we discuss the design and performance of these foops. Special attention are paid to the error analysis of the system behavior under small deviation of relevant parameters. The differences under open and closed loop condition are demonstrated. Only the dipole oscillation of the bunch is considered, the quadrupole oscillation suppression will be covered in future report. Whenever numerical example is need, the BNL AGS Booster is used as an example. Same analysis can be applied to synchrotion during transition crossing where wide variation of system parameter is expected.

$A$ very useful introduction to the analysis of RF feedback loops can be found in the paper by D. Boussard [1], the design of Booster RF system is taken from Booster Design Manual [2] and also from communication with M. Brennan [3].

* Work performed under the auspices of the U.S. Department of Energy 


\subsection{The Frequency Program}

A simplest accelerator RF system consists of cavities driven by oscillators. The frequency of oscillators is determined from the magnetic field by the following frequency program,

$$
\omega_{r f}=\frac{h c}{R} \frac{B}{\left(B^{2}+\left(\frac{E_{0}}{c \rho}\right)^{2}\right)^{1 / 2}}
$$

where

$h$ is the harmonic number, (in Booster, $h$ is 3 ).

$c$ is the velocity of light, $3 \times 10^{8} \mathrm{~m} / \mathrm{sec}$.

$R$ is the mean radius of the accelerator, ( $R$ is $32.114 m$ ).

$B$ is the magnetic field, (for proton, $B_{i n j}=0.156 \mathrm{Tesla}$, and $B_{\text {eje }}=0.546 \mathrm{Tesla}$ ).

$E_{0}$ is the rest energy of proton, it is $0.938 \mathrm{Gev}$.

$\rho$ is the radius of curvature of the magnets, $(13.751 \mathrm{~m})$.

From the measurement of the magnetic field the frequency program generates a voltage, which drives the oscillators to generate a frequency, and the cavities are in turn driven by the oscillators. The accelerating frequency of the cavity gap voltage, $\omega_{r f}$, is presumably following the frequency of the oscillators. All elements, especially the oscillators and cavities, will however inevitably introduce errors and delays.

Any error in the accelerating frequency will generate beam radial deviation from the equilibrium position that is shown in

$$
\frac{\Delta R}{R}=\frac{\gamma^{2}}{\gamma_{t r}^{2}-\gamma^{2}} \frac{\Delta \omega_{r f}}{\omega_{r f}}=-\frac{1}{\eta \gamma_{t r}^{2}} \frac{\Delta \omega_{r f}}{\omega_{r f}}
$$

where $\boldsymbol{\gamma}$ and $\boldsymbol{\gamma}_{t r}$ are the usual relativistic parameter and its value at the transition (in Booster, $\gamma_{t r}$ is 4.88 ), respectively, and $\eta$ is the frequency slip factor,

$$
\eta=\frac{1}{\gamma_{t r}^{2}}-\frac{1}{\gamma^{2}}
$$

During the acceleration, $\eta$ varies from -0.65 to -0.1 in the Booster. Therefore roughly speaking, a 0.1 percent frequency error will yield radial deviations from $0.2 \mathrm{~cm}$ to $1.34 \mathrm{~cm}$. Meanwhile, the oscillator alone may generate more than a 0.1 percent error. It is therefore necessary to apply radius feedback regulations to compensate the possible frequency error.

\subsection{Beam Transfer Function}

To regulate the beam, or st know the beam transfer function. Although the complete beam transfer functions from we driving. frequency to the phase and radial positions are highly nonlinear and sophisticated, a simple linearized model of the beam small signal dynamic can be obtained, that is shown in Fig.1, 


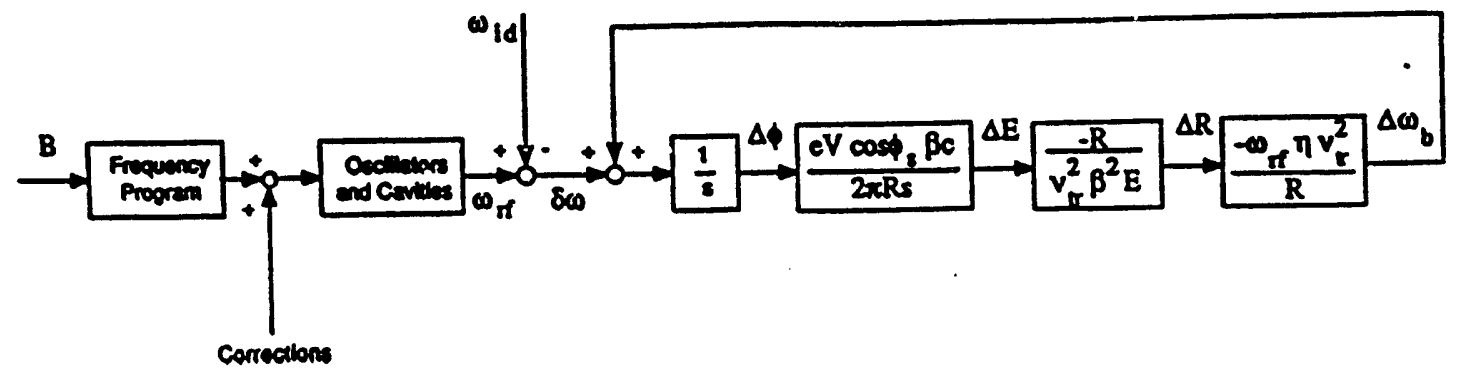

Fig.1. Beam Dynamic Model

where $s$ is the Laplace operator, in frequency domain it can be replaced by $j \omega$, while in time domain, it represents a derivative operation. The initial conditions are disregarded momentarily, and will be discussed later. The variable $\delta \omega$ is the difference between the accelerating frequency $\omega_{r f}$ and the ideal beam frequency $\omega_{i d}$. Other variables are listed as follows.

$V$ is the crest gap voltage, which is independently preprogrammed.

$\phi_{2}$ is the stable phase, it is determined by the derivative of the magnetic field and the gap voltage $V$.

$\beta$ is the ratio of the particle velocity $v$ and the light velocity $c$, and $E$ is the total energy of the particle.

$\Delta \phi$ and $\Delta R$ are the beam phase and radial deviations from the equilibrium state, respectively.

$\Delta E$ and $\Delta \omega_{b}$ are the beam energy and frequency deviations, respectively.

The details of this model will be discussed as the follows.

A phase deviation $\Delta \phi$ yields an energy deviation per revolution of $e V \cos \phi, \Delta \phi$. Since $\frac{\beta c}{2 \pi R}$ is the turns per unit time, the energy deviation per unit time, due to $\Delta \phi$, is

$$
s \Delta E=\frac{e V \cos \phi, \beta c}{2 \pi R} \Delta \phi
$$

This energy deviation $\Delta E$ corresponds to a beam radial deviation

$$
\Delta R=-\frac{R}{\gamma_{t r}^{2} \beta^{2} E} \Delta E
$$

From equation (2), the beam frequency deviation is

$$
\Delta \omega_{b}=-\frac{\omega_{r f} \eta \gamma_{l r}^{2}}{R} \Delta R
$$

A lag of the beam arriving at the cavity is equivalent to an advance of the driving frequency phase. Therefore the total phase difference between the RF frequency and the beam frequency shown at the cavity is the sum of the beam phase deviation $\Delta \omega_{b}$ and the driving frequency 
deviation-

Sw. This makes the loop ciosed, as shown in Fig.1.

Finaily, we note that after an integration, represented by $\frac{1}{s}$, the frequericy difference becomes a phase deviation $\Delta \phi$. This process is implemented in the low level RF system by a phase detector. The driving frequency and the beam frequency are measured by a cavity gap voltage detector, and by a beam pick-up electrodes (PUE), respectively.

We note that the real beam frequency should be the vector sum of the ideal beam frequency and the beam frequency deviation, which is not shown explicitly in Fig.1.

By inspecting the model in Fig.1, one obtains

$$
\begin{gathered}
s \Delta \phi=-\frac{\omega_{r f} \eta \gamma_{l r}^{2}}{R} \Delta R+\delta \omega \\
s \Delta R=-\frac{e V \cos \phi_{\bullet} c}{2 \pi \beta \gamma_{l r}^{2} E} \Delta \phi
\end{gathered}
$$

To simplify the linear model, we define

$$
\begin{aligned}
& a=-\frac{\omega_{r f} \eta \gamma_{l_{r}}^{2}}{R} \\
& b=-\frac{e V \cos \phi_{c} c}{2 \pi \gamma_{l r}^{2} \beta E}
\end{aligned}
$$

Thus the equation (7) can be written as

$$
\begin{gathered}
s \Delta \phi=a \Delta R+\delta \omega \\
s \Delta R=b \Delta \phi
\end{gathered}
$$

where $a$ and $b$ are the machine parameters considered to be constant during the damping period.

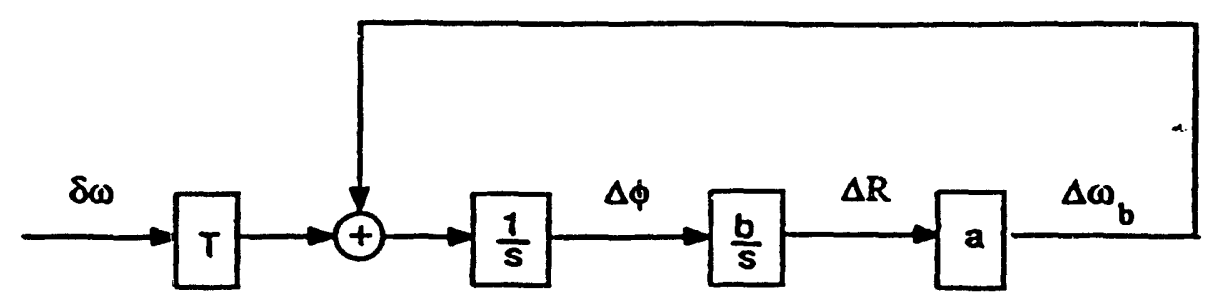

Fig.2. Simplified Beam Dynamic Model

The simplified model is shown in Fig.2, where the energy deviation $\Delta E$ is not explicitly shown. Note that we add a box $T$, which stands for the oscillators and cavities, representing that all regulations and controls will go through these elements. If the errors from them is neglected, we may let $T=1$. In the model, we note that $\eta$ will change sign at the transition, 
so is $a$. In that case, the synchrotron phase $\phi_{0}$ in $b$ has to be changed to $\pi-\phi_{0}$ in order to keep the stable oscillation.

The transfer function from the input frequency perturbation $\delta \omega$ to the radial and phase deviations, $T_{1}$ and $T_{2}$, can therefore be obtained by eliminating variable $\Delta \phi$ and $\Delta R$ in the equation (10), respectively, giving

$$
\begin{aligned}
& \Delta R=T_{1} \delta \omega=\frac{b}{s^{2}-a b} \delta \omega=\frac{b}{s^{2}+\Omega_{s}^{2}} \delta \omega \\
& \Delta \phi=T_{2} \delta \omega=\frac{s}{s^{2}-a b} \delta \omega=\frac{s}{s^{2}+\Omega_{s}^{2}} \delta \omega
\end{aligned}
$$

where

$$
\Omega_{s}=(-a b)^{1 / 2}=\left(-\frac{\omega_{r f} e V \eta \cos \phi_{s} c}{2 \pi R \beta E}\right)^{1 / 2}
$$

is the synchrotron oscillation frequency.

We note that once the magnetic field and the RF crest gap voltage have been programmed, then the accelerating frequency, stable phase, and many other parameters are determined. Among them, $a$ and $b$ are the ones explicitly contribute to the beam transfer function, and therefore to the beam controls as well. We plot $f_{f}=\Omega_{0} / 2 \pi, a$, and $b$ of the Booster during the acceleration in Fig.3. It should be noted that these variable span a wide range during the acceleration. Therefore, the performance and stability of the feedback loops should be examined carefully for each parameter extremes of $a$ and $b$, especially if the transition energy crossing is involved.

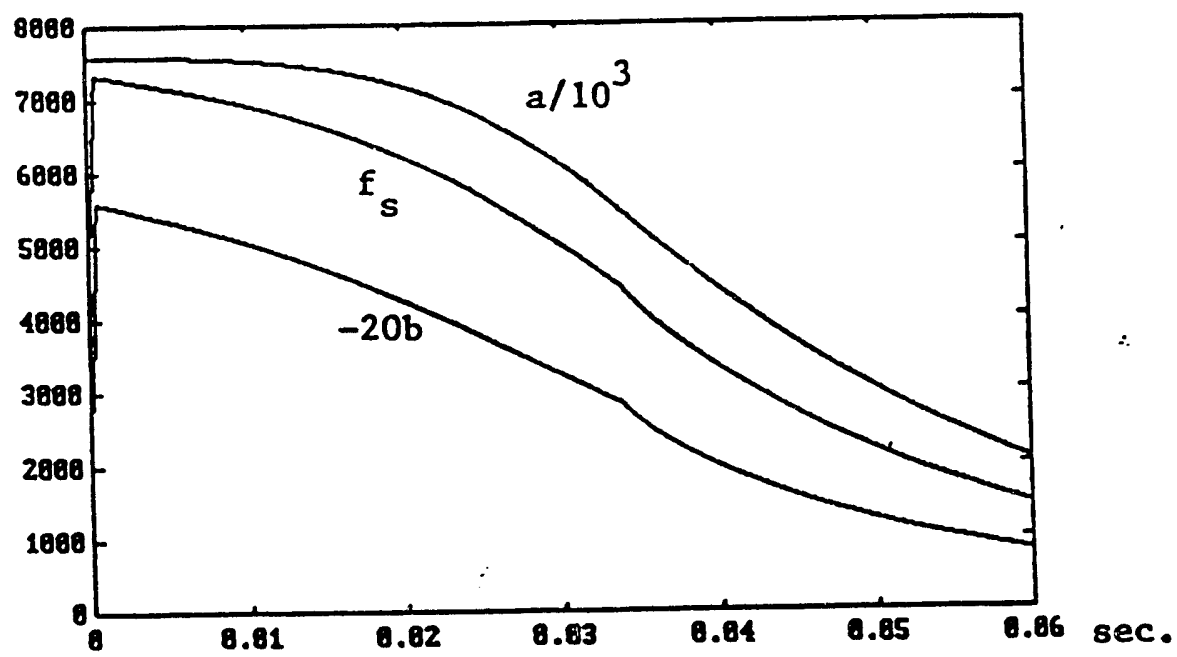

Fig.3. Booster Parameters $f_{,}, a$, and $b$ During Acceleration. 


\subsection{Phase Control Loop}

By inspecting the diagram in Fig.2 and the transfer functions in equations (11) and (12), we find that the following problems have to be solved.

i) The fundamental mode of the beam transfer functions, which is determined by the denominator of the transfer functions, is oscillatory without damping. All the non-zero initial conditions and any deviation from the equilibrium state will induce their own oscillar tions. The summation of these oscillations determines the beam longitudinal motion, which is also oscillatory. More importantly, if there is a harmonic from any disturbances whose frequency coincides with the synchrotron oscillation frequency, then the emittance will blow-up.

ii) All the error in the block $T$, which represents the oscillators and cavities, are directly responsible to the deviations in the beam motion. These errors are not corrected.

ii) The disturbances may also come from the deviations of the beam itself, in the forms of radial position, phase, or frequency. Such kind of error, either from the design and manufacture inaccuracy or from the outside interference, is inevitable. These errors are also not corrected.

One solution to these problems is to introduce feedback regulations. Local loops encircling the oscillators and cavities may improve their performance to certain extent, but it is not helpful to the problems in i) and iii). Therefore feedback loops that encircle either the beam phase or radial deviations have to be introduced. Since these two variables are related, see equation (7), therefore we may assume that either one should help. A direct feedback from the radial deviation with a simple amplifier will not change the oscillatory mode, as can be seen from the transfer function $T_{1}$ in equation (11). Therefore we consider first a phase feedback. The phase deviation $\Delta \phi$ is taken from a phase detector, which compares the RF frequency from the cavity gap voltage detector, and the beam frequency from the PUE's. The signal then is sent to the oscillators for correction. This is shown in Fig.4, where $G_{1}$ represents the feedback network.

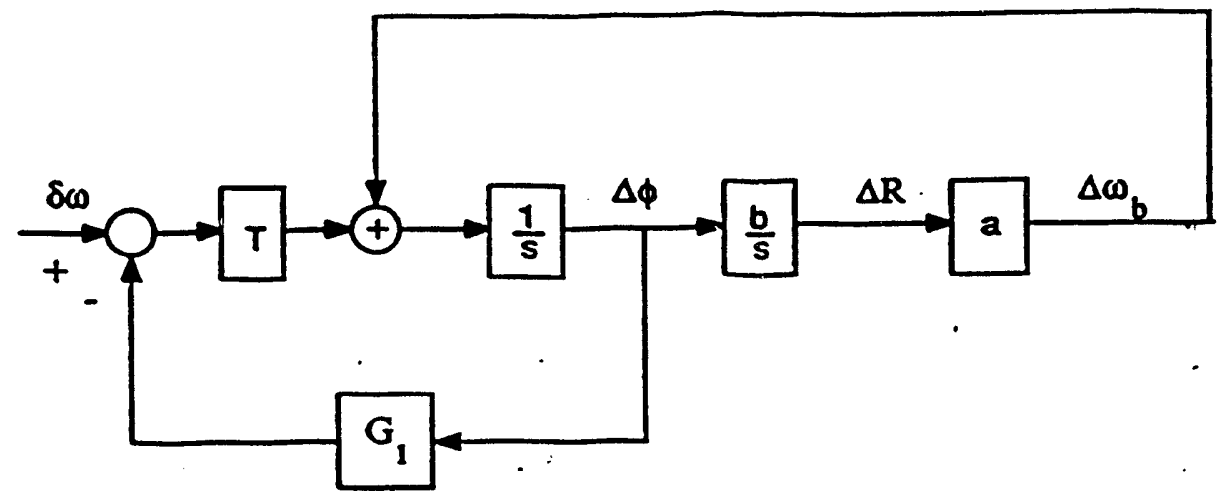

Fig.4. Phase Control Loop

In the next, we shall discuss the stability, system performance, and possible improvements related to the phase loop. 
With the phase feedback, the transfer functions from $\delta \omega$ to $\Delta R$ and $\Delta \phi$ with the phase loop are derived as follows.

$$
\begin{aligned}
& \Delta R=T_{3} \delta \omega=\frac{T_{1}}{1+G_{1} T_{2}} \delta \omega=\frac{b}{s^{2}+k_{1} s+\Omega_{a}^{2}} \delta \omega \\
& \Delta \phi=T_{1} \delta \omega=\frac{T_{2}}{1+G_{1} T_{2}} \delta \omega=\frac{s}{s^{2}+k_{1} s+\Omega_{a}^{2}} \delta \omega
\end{aligned}
$$

where we let $G_{1}=k_{1}$, a simple amplifier, which provides damping. For the second order system such as $T_{X}$ and $T_{Y}$, the term $\Omega_{\text {g }}$ is called the natural frequency. In this model, the natural frequency equals the synchrotron oscillation frequency. The damping ratio is defined as

$$
\varsigma=\frac{k_{1}}{2 \Omega_{s}}
$$

If $S=1$, the system is critically damped. Sometimes, one uses $\tau=\frac{2}{k_{1}}$ to estimate the system time constant. This is useful for the damping ratio smaller than 1 . If $S$ is larger than 1 , then the system is overdamped, the real time constant might be much longer than $\tau$.

For the stability analysis, we use Root Locus method. Instead of studying the amplitude and phase transmission properties along with the frequency variation, such as Bode, Nichols and Nyquist methods, the root locus method finds the closed loop poles directly from the open loop poles and zeros. The variable taken in the root locus method is the loop gain, which now is largely deternined by the compensator adjustment.

The clcsed loop pole, or root locus start from the open loop poles, which correspond to zero loop gain. They move when the gain increases, and finally with infinite gain they reach the open loop zeroes, some of which might be at the infinity. With these closed loop poles, one makes the judgement of the stability, finds the oscillation frequency, the damping or antidamping rate, and one determines the dominant poles as well.

From Fig.3, we know that the synchrotron oscillation frequency at Booster varies from $7.3 \mathrm{kHz}$ to $1.46 \mathrm{kHz}$. The design of the phase loop must accommodate the whole range. In the following analysis, a typical synchrotron oscillation frequency $5 \mathrm{kHz}$ is used for illustration.

For the phase loop, the open loop transfer function is

$$
T_{o 1}=G_{1} T_{2}=\frac{k_{1} s}{s^{2}+\Omega_{s}^{2}}
$$

In Fig.5, we show the root locus of this system. In the plot the closed loop poles corresponding to zero loop gain, i.e., the open loop poles in fact, are marked by ' $x$ ', and other closed loop poles are marked by' 0 ', which correspond to a certain loop gain. The open loop poles in Fig.5 are on the imaginary axis, representing an oscillation mode. When .the gain increases, the poles move to the left. They meet at the real axis, where $\$$ equals 1 , and then separate again. The one on the right branch then is called the dominant pole, due to its proximity to the $s$ plane origin. It is clear that the stability is always guaranteed. 


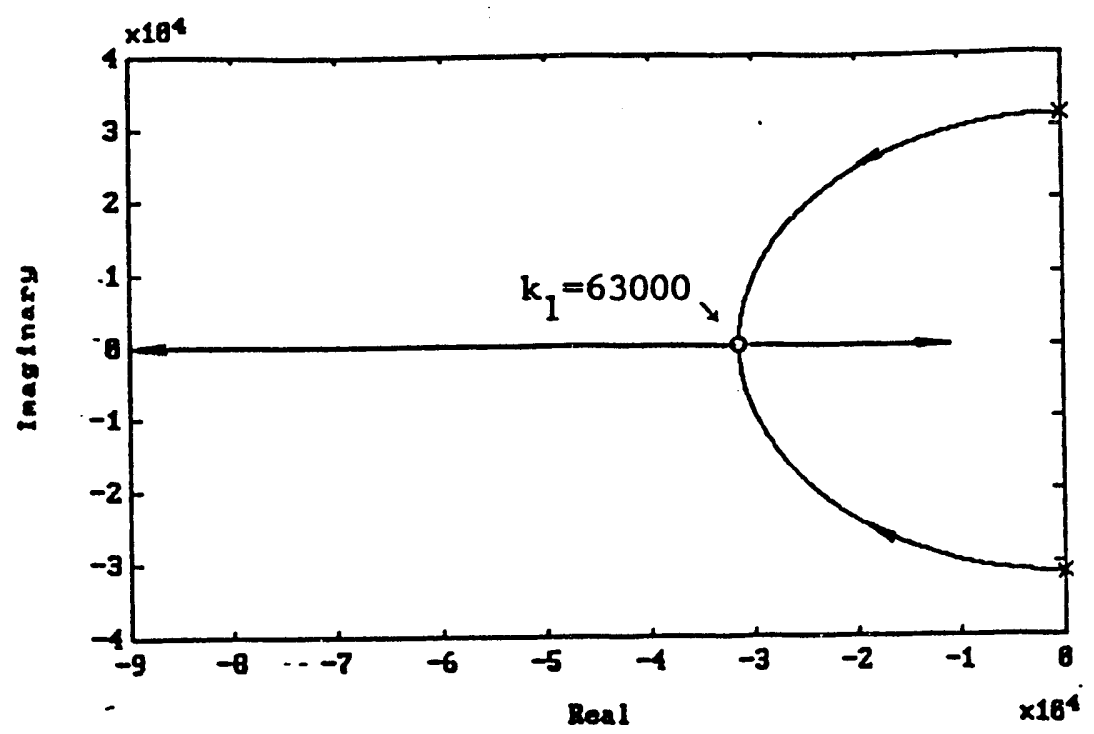

Fig.5. Root Locus of Phase Control Loop

In choosing $k_{1}$, we need the system to be properly damped. The fast system settling down with some overshoot conventionally requires $S=0.707$. To avoid overshoot, the critical damping might be an acceptable choice. From equation (16), we find that $k_{1}=63 \cdot 10^{3}$ would give rise to a critical damping. If $k_{1}$ is larger than that, the dominated pole will move close to the origin, that implies longer settling time. For instance, $k_{1}=10^{6}$ will let the dominant pole move to $-10^{3}$ on the real axis, resulting in a time constant of approximately $1 \mathrm{~ms}$. This is certainly too long. The gain $k_{1}$ therefore may be chosen from a range of $2 \cdot 10^{4}$ to $2 \cdot 10^{5}$ for Booster.

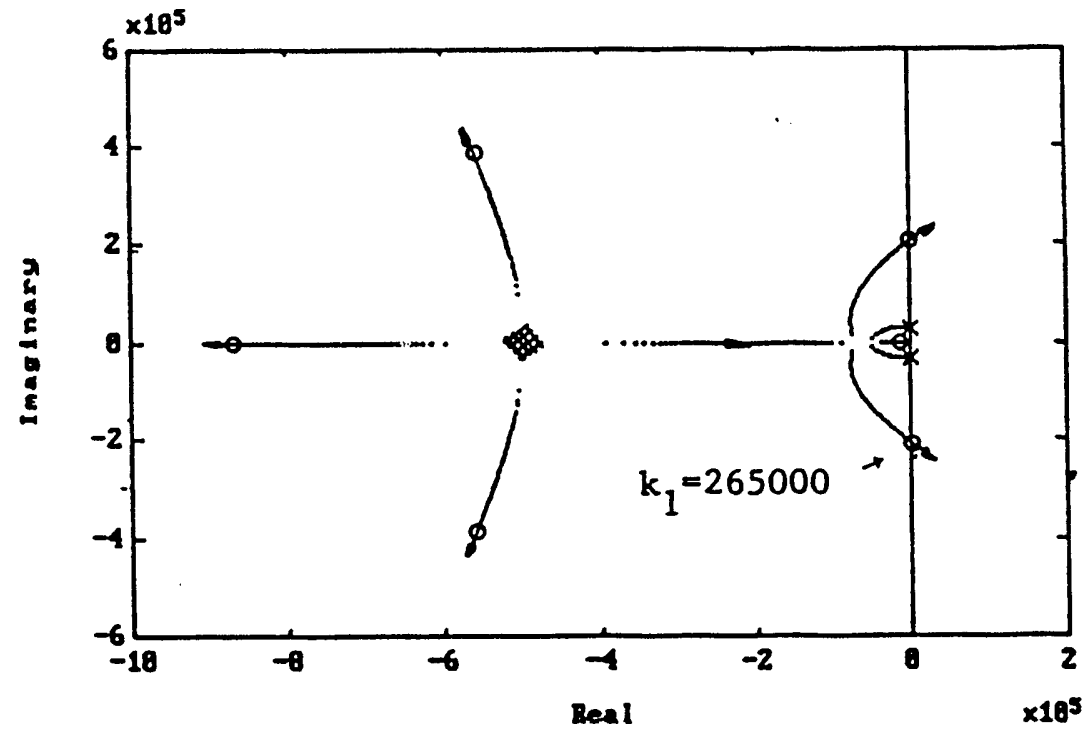

Fig.6. Root Locus of Phase Control Loop with Delays

In reality, we must also consider the cable time delay, and the limited bandwidth of the oscillators, amplifiers, and cavities. For simplicity, the time delay can be approximated as a 
low pass network as follows.

$$
e^{-r s} \simeq \frac{1}{\tau s+1}
$$

where $T$ is the delay time. With this delay, the open loop transfer function becones

$$
T_{02}=\frac{k_{1} s}{\left(s^{2}+\Omega_{c}^{2}\right)(\tau s+1)}
$$

For Booster, with four $2 \mu s$ delay factors in series, a gain of $265 \cdot 10^{3}$ causes instability, as shown in Fig.6. The parameters taken in this example are not very far from the real RF system of the AGS Booster. These delays should be properly estimated and considered in the design.

\subsection{Performance Analysis}

We first note that by introducing the phase loop, the beam oscillation can be properly damped. Using the transfer function (14), we plot the responses of $\Delta R$ due to a step $\delta \omega$, with different phase feedback gains, shown in Fig.7. It can be noticed that the choice of the gain $k_{1}$ of $63 \cdot 10^{3}$, or $63 \mathrm{kHz} / \mathrm{rad}$, gives rise to a critically damped response. The system settles down within $200 \mu s$.

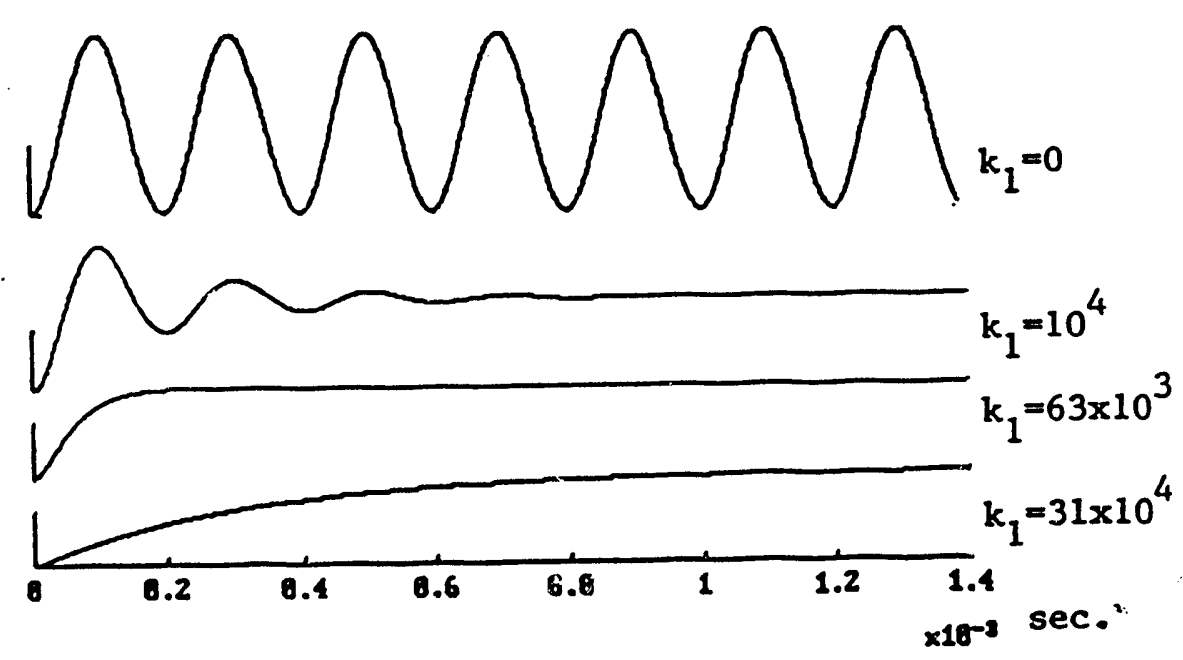

Fig.7. Radial Responses $\Delta R$ due to a Step Frequency Error With Phase Control Loop

Secondly, the transient responses for the possible beam disturbances are concerned. These disturbances might be in the forms of the phase, energy, radial position, or frequency. As shown in Fig.1, the energy, the radial position, and the frequency deviations are directly related by constants. Therefore, without loss of generality, we simulate the beam disturbance as the phase and frequency deviation steps, as shown in Fig.8. 


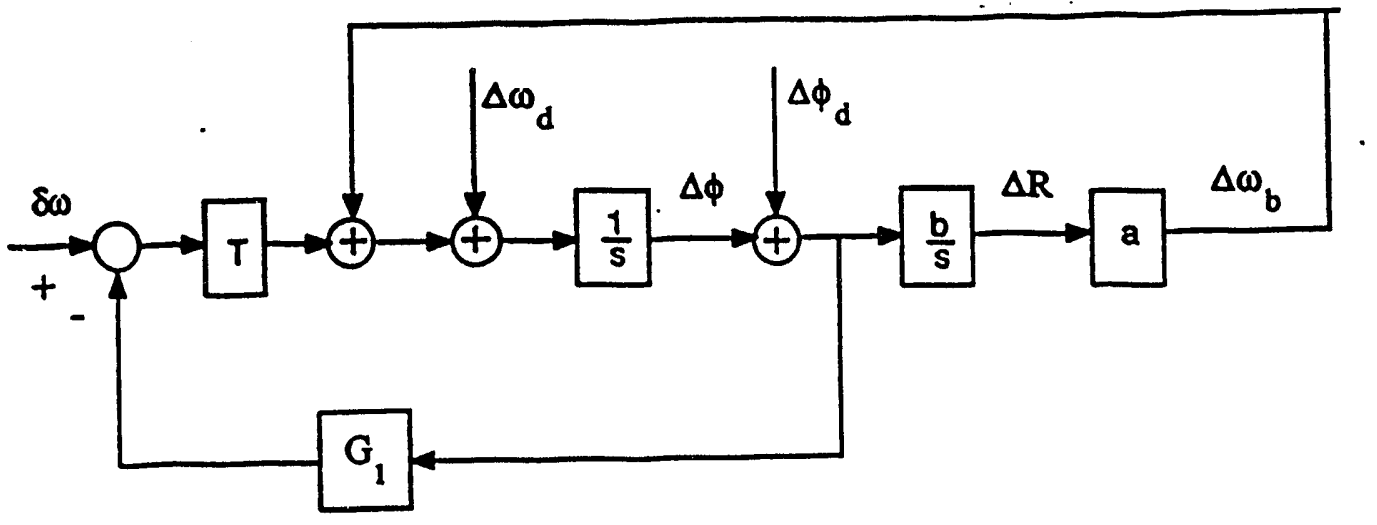

Fig.8. Phase Control Loop with Phase and Frequency Disturbances

With the step perturbation $\frac{1}{s}$, the radial responses are shown in Fig.9, following the relations given in equations (20) and (21).

$$
\begin{gathered}
\Delta R=T_{3} \Delta \omega_{d}=\frac{b}{s^{2}+k_{1} s+\Omega_{s}^{2}} \Delta \omega_{d}=\frac{b}{s^{2}+k_{1} s+\Omega_{s}^{2}} \frac{1}{s} \\
\Delta R=T_{5} \Delta \phi_{d}=s T_{3} \Delta \phi_{d}=\frac{b s}{s^{2}+k_{1} s+\Omega_{d}^{2}} \Delta \phi_{d}=\frac{b s}{s^{2}+k_{1} s+\Omega_{d}^{2}} \frac{1}{s}
\end{gathered}
$$

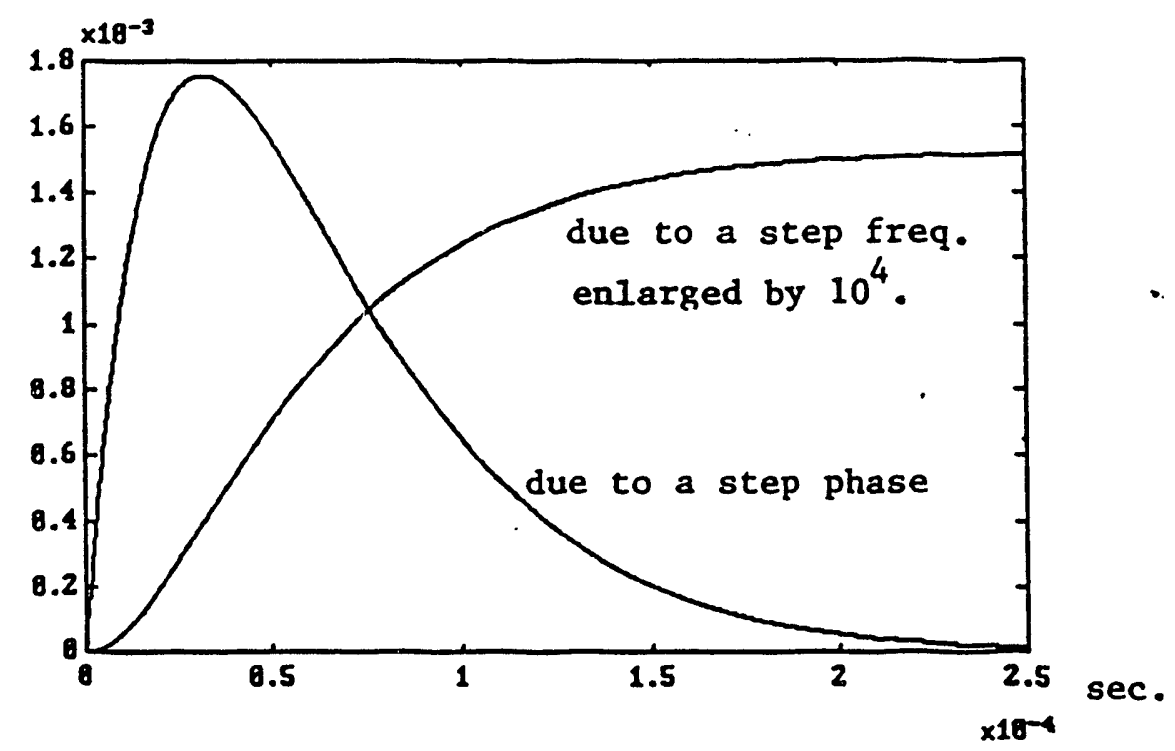

Fig.9. Radial Responses for Step Phase and Frequency Perturbations With Phase Control Loop 
The response of the radial deviation due to the phase disturbance is the derivative of that due to the frequency disturbance. With the phase feedback gain of $63 \mathrm{kHz} / \mathrm{rad}$, they are all critically damped.

Finally, we have to know the residual error. Without phase loop, any response to a nonzero input will keep oscillating without damping. With a proper phase loop, we have the following residual error for a unit step of $\delta \omega$,

$$
\begin{gathered}
\lim _{t \rightarrow \infty} \Delta R=\lim _{t \rightarrow 0} s T_{3} \cdot \frac{1}{s}=\frac{b}{\Omega_{s}^{2}}=-\frac{1}{a} \\
\lim _{t \rightarrow \infty} \Delta \phi=\lim _{t \rightarrow 0} s T_{4} \cdot \frac{1}{s}=0
\end{gathered}
$$

The equation (23) implies that the residual error of the phase deviation due to a step frequency disturbance is zero, however equation (22) shows that the residual error of the radial deviation is $-\frac{1}{a}$.

Comparing equations (2) and (8), it is clear that the residual error of $\Delta R$ due to the frequency disturbance is not affected by the phase loop. Therefore by increasing the phase feedback gain $k_{1}$, the damping ratio can be changed, but the residual error. of the radius will not be afiected.

\section{S Further Performance Improvement}

By using a simple amplifier as the phase feedback the damping can be achieved, however the following problems remain,

i) Although the system is damped by the phase loop, the absolute value of the residual radial deviation due to accelerating frequency errors has not been improved, it might still be too large to be acceptable.

ii) We also note that the damping may take too long, for example the cases shown in Figs. 7 and 9 the damping take about $200 \mu s$. With a certain damping ratio $S$ the damping time, or the damping rate, is determined by the system natural frequency. Since the phase feedback with a simple amplifier cannot change the system natural frequency, the solution proposed so far cannot address this issue.

The cure to these two problems can be made by modifying $G_{1}$. Let

$$
G_{1}=k_{1}+\frac{k_{2}}{s}
$$

which means that in parallel to the phase feedback amplifier, we add an integrator with a static gain of $k_{2}$. This type of feedback is called the proportional and integral feedback.

The transfer functions now become

$$
\begin{aligned}
& \Delta R=T_{6} \delta \omega=\frac{q_{1}}{1+G_{1} T_{2}} \delta \omega=\frac{b}{s^{2}+k_{1} s+k_{2}+\Omega_{s}^{2}} \delta \omega \\
& \Delta \phi=T_{7} \delta \omega=\frac{T_{2}}{1+G_{1} T_{2}} \delta \omega=\frac{s}{s^{2}+k_{1} s+k_{2}+\Omega_{s}^{2}} \delta \omega
\end{aligned}
$$

Comparing the equation (25) and (26) to the equation (14) and (15), the following differences are noticed. 
i) If one choose $k_{2} \gg \Omega_{\theta}^{2}$, then the residual radial error can be greatly reduced. Specifically, it is reduced by a factor of $\frac{k_{2}+\Omega_{e}^{2}}{\Omega_{f}^{2}}$.

ii) Also the natural frequency is increased from $\Omega_{s}$ to $\left(k_{2}+\Omega_{s}^{2}\right)^{1 / 2}$, which means that with a same damping ratio $\zeta$, the system response can be much faster.

ii) If $k_{1}$ is not changed, then the damping ratio is decreased. In order to keep the same damping, we have to increase $k_{1}$.

By choosing $k_{2}=2 \cdot 10^{\circ}$, the step responses of $\Delta R$ are shown again in Fig.10. Comparing to the responses in Fig.7, we note that the damping ratio is smaller for the same $k_{1}$, while the responses are faster.
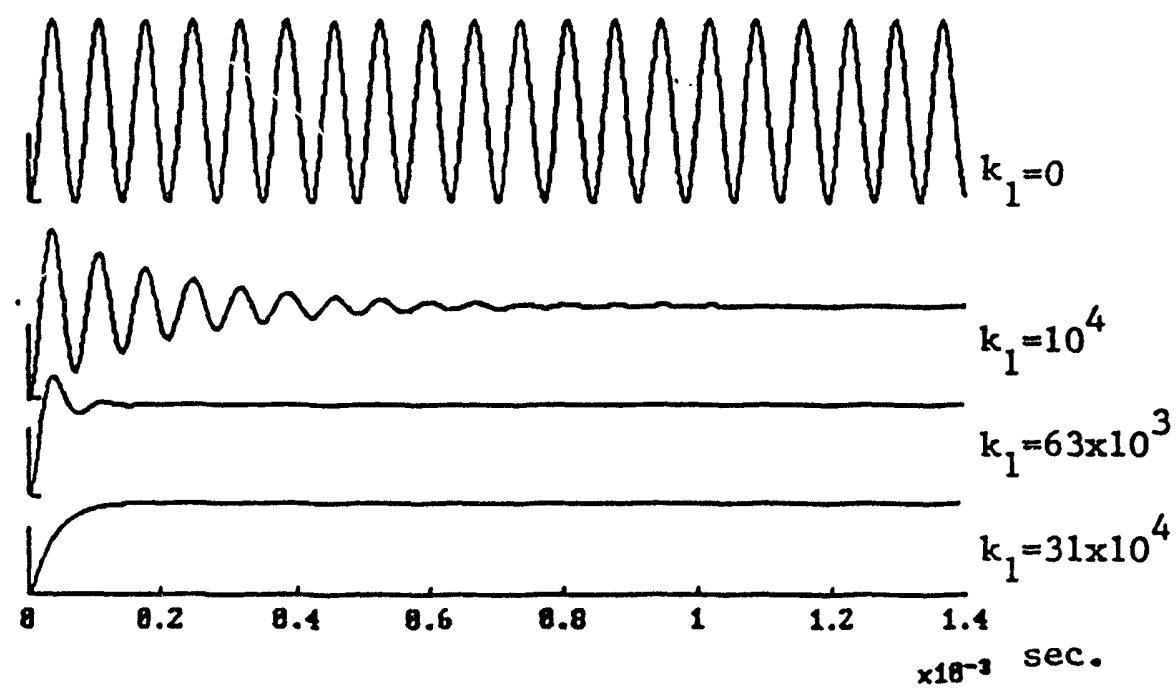

Fig.10. Radial Responses $\Delta R$ due to a Step Frequency Error With Proportional and Integral Phase Feedback, $k_{2}=7 \cdot 10^{8}$

For the stability analysis, we open the integrator branch in $G_{2}$, then the open loop transfer function is

$$
T_{03}=\frac{k_{2}}{s} T_{1}=\frac{k_{2}}{s^{2}+k_{1} s+\Omega_{s}^{2}}
$$

Note the cancellation of $s$ between the denominator of $\frac{k_{2}}{s}$ and the numerator of $T_{4}$. The root locus shown in Fiz.11a indicates that the stability is guaranteed. If the phase delay due to the cables, oscillators, filters, and cavities are included, then the root locus can be seen in Fig.11b. 


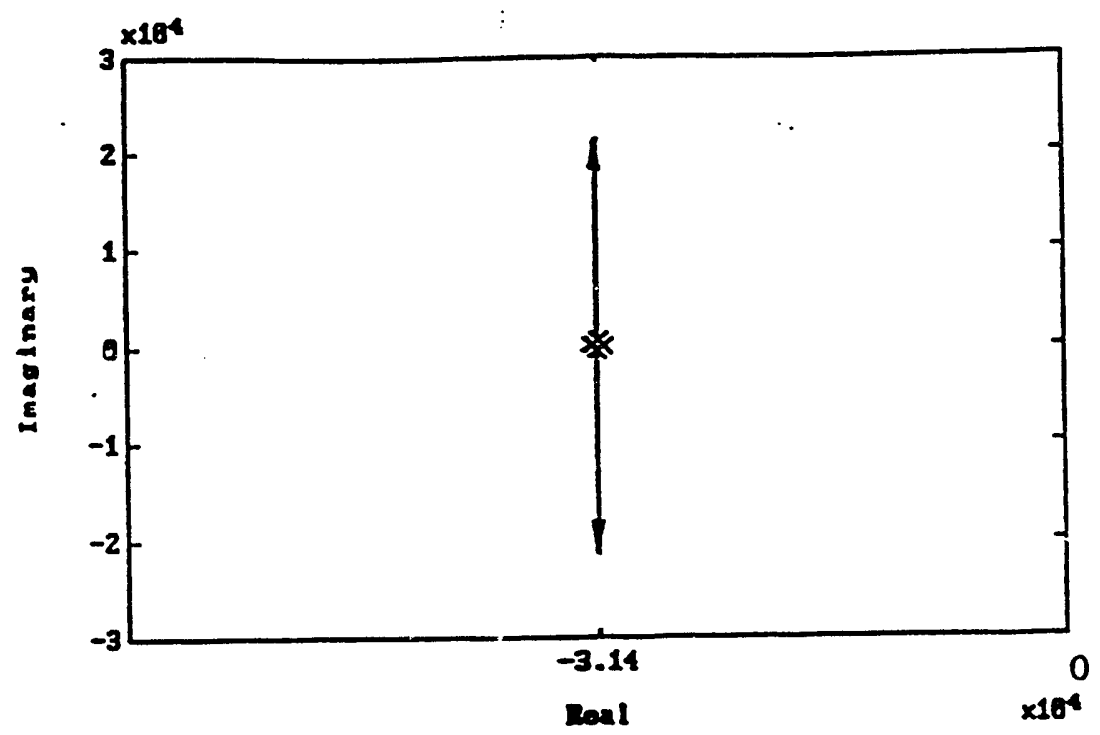

Fig.11a. Root Locus of Phase Control Loop with Proportional and Integral Feedbark

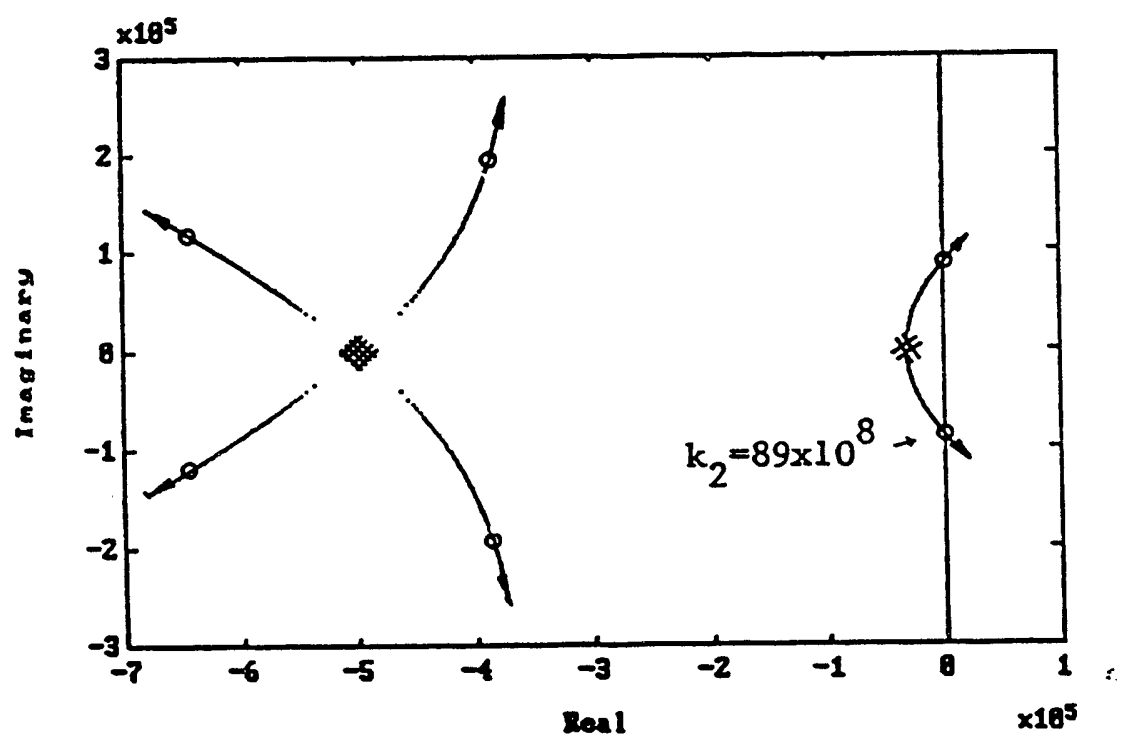

Fig.11b. Root Locus of Phase Control Loop with Proportional and Integral Feedback with Delays

Where it is clear that when $k_{2}$ becomes large, the closed poles will move to the right and across the imaginary axis, causing instability. For four delay factors each with delay time $2 \mu s, k_{2}$ cannot be larger than $89 \cdot 10^{8}$.

\subsection{Radial Control Loop Plus Phase Control Loop}

A more straightforward approach to realize the same effort as the proportional and integral type phase feedback is the radial plus phase feedback, which is shown in Fig.12. 


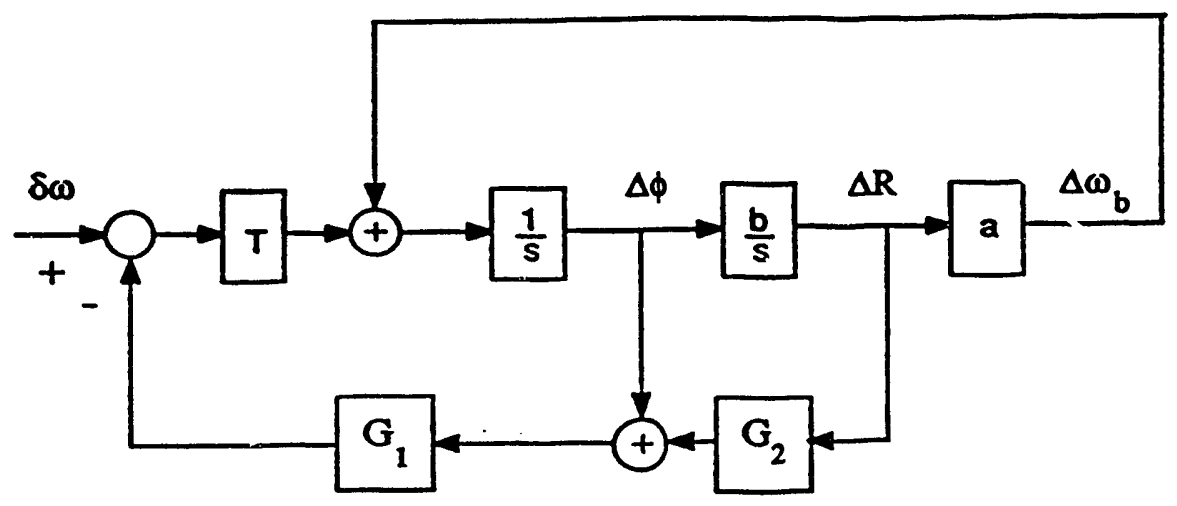

Fig.12. Radial Control Loop Plus Phase Control Loop

The trinsfer furictions then become,

$$
\begin{aligned}
& \Delta R=T_{8} \delta \omega=\frac{b}{s^{2}+k_{1} s+b k_{1} G_{2}+\Omega_{\theta}^{2}} \delta \omega \\
& \Delta \phi=T_{9} \delta \omega=\frac{s}{s^{2}+k_{1} s+b k_{1} G_{2}+\Omega_{\theta}^{2}} \delta \omega
\end{aligned}
$$

To simplify the analysis, we let $G_{2}=k_{3}$. Since usually $b k_{1} k_{3} \gg \Omega_{6}^{2}$, the residual error of $\Delta R$ due to a unit step of $\delta \omega$ is now about $\frac{1}{k_{1} k_{3}}$, which is not affected by the variations of $a$ or $b$ during the acceleration. In this sense, the radial feedback is somewhat superior than the proportional and integral type phase feedback.

The rest of the analysis can be done in a similar manner to the proportional and integral type phase feedback, and it will not be repeated. Note that since $\Delta R=\frac{b}{s} \Delta \phi$, then the relation between the radial plus phase feedback and the proportional and integral phase feedback can be represented by $k_{2}=b k_{3} k_{1}$. For instance, in the example shown in Fig.10, we let $k_{2}=7 \cdot 10^{9}$. If we choose $k_{1}=63 \cdot 10^{3}$ and take $b=-150$, then this is equivalent to $k_{3}=-741$.

For further illustration, let us consider the example of $5 \mathrm{kHz}$ synchrotron oscillation and therefore $\Omega=314 \cdot 10^{2}$. Then the damping ratio is only 0.35 and the time constant is approxim to roughly $\frac{1}{k_{1} k_{3}}$, i.e., from $16 \cdot 10^{-8}$ to $2 \cdot 10^{-8}$. On the other hand, if a better damping is desired one inây c'soose $k_{1}=179 \cdot 10^{3}$ and $k_{3}=260$, that gives rise to $S=1$. The time constant is reduced to about $11 \mu s$ and the residual error keeps the same, i.e., $2 \cdot 10^{-8}$.

To reduce further the residual error of $\Delta R$ due to a $\delta \omega$, we consider the integrator type radial feedback, and let

$$
G_{2}=\frac{k_{4}}{s}
$$


Then we have

$$
\begin{gathered}
\Delta R=T_{10} \delta \omega=\frac{b}{s^{2}+k_{1} s+b k_{1} \frac{k_{4}}{s}+\Omega_{s}^{2}} \delta \omega \\
=\frac{b s}{s^{3}+k_{1} s^{2}+\Omega_{s}^{2} s+b k_{1} k_{4}} \delta \omega
\end{gathered}
$$

The residual error of $\Delta R$ due to a step $\delta \omega$ becomes

$$
\lim _{t \rightarrow \infty} \Delta R=\lim _{l \rightarrow 0} s T_{10} \cdot \frac{1}{s}=0
$$

i.e., a step RF frequency error induces eventually a zero radial error.

To plot the root locus, we find the open loop transfer function as

$$
T_{O 4}=\frac{k_{4}}{s} \frac{b k_{1}}{s^{2}+k_{1} s+\Omega_{s}^{2}}
$$

The root locus shown in Fig.13a indicates that the gain $k_{4}$ is limited due to the additional open loop pole at the origin, which is posed by $G_{2}$ in the equation (30). The stability requires $k_{1}$ to be smaller than $63 \cdot 10^{5}$. If we consider again the four $2 \mu s$ delays in series, then the root locus are shown in Fig.13b. The stable gain now reduces to $42 \cdot 10^{5}$, slightly smaller than the original limit.

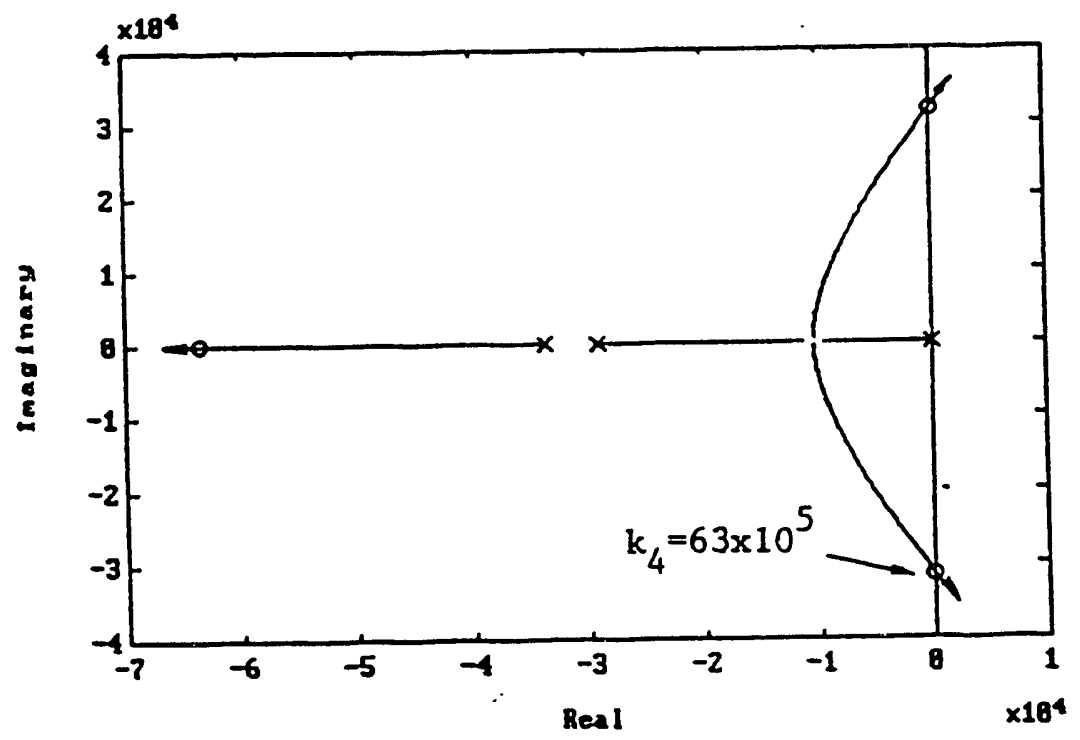

Fig.13a. Root Locus of Radial Plus Phase Control Loops, without Delay 


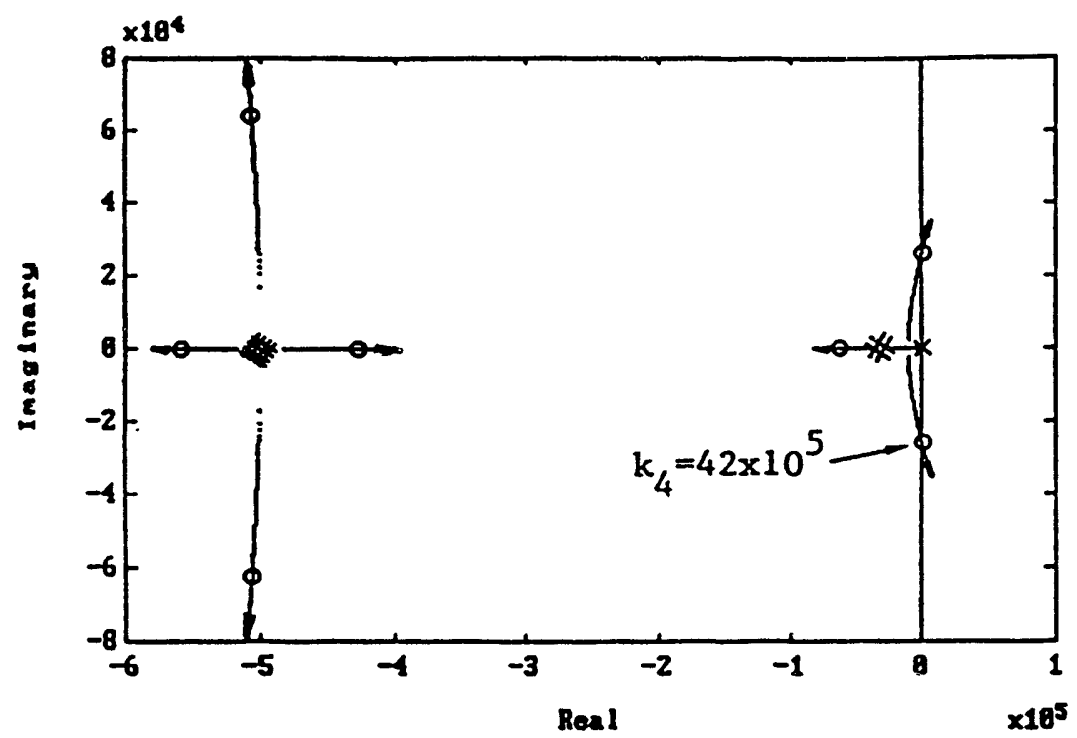

Fig.13b. Root Locus of Radial Plus Phase Control Loops, with Delays

\subsection{References}

[1] D. Boussard, "An Elementary Presentation of the PS Beam Control System," MPS/SR/Note 73-10, 1973.

[2] Booster Design Manual, October, 1988, BNL.

[3] M. Brennan, Private Communication.

[4] W. T. Weng, "Fundamentals - Longitudinal Motion," AIP Conference Proceedings, 184, pp. 243-287, 1989.

\section{DISCLAIMER}

This report was prepared as an account of work sponsored by an agency of the United States Government. Neither the United States Government nor any agency thereof, nor any of their employees, makes any warranty, express or implied, or assumes any legal liability or responsibility for the accuracy, completeness, or usefulness of any information, apparatus, product, or process disclosed, or represents that its use would not infringe privately owned rights. Reference herein to any specific commercial product, process, or service by trade name, trademark, manufacturer, or otherwise does not necessarily constitute or imply its endorsement, recommendation, or favoring by the United States Government or any agency thereof. The views and opinions of authors expressed herein do not necessarily state or reflect those of the United States Government or any agency thereof. 

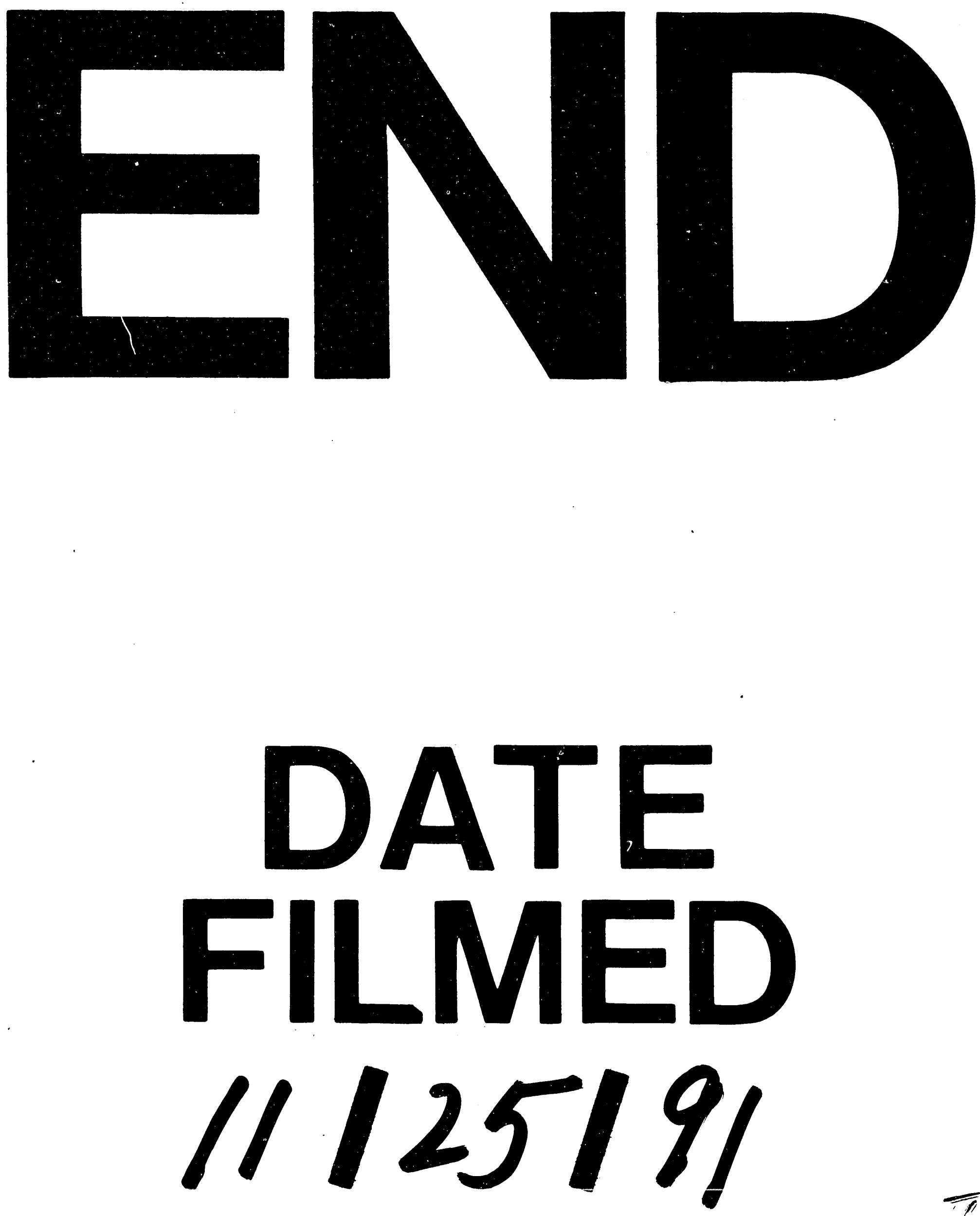
